
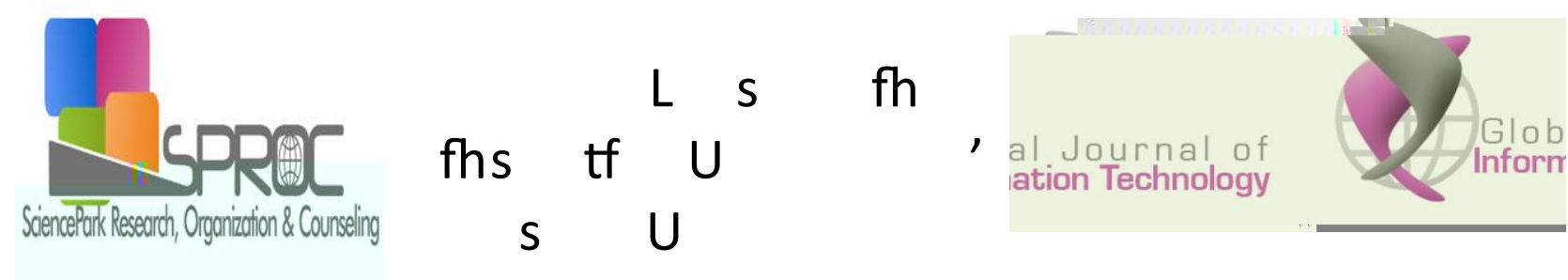

www.gjit.eu

\title{
Conceptual analysis studies in Turkish tests and suggestions to solve the computer network terms lexicon
}

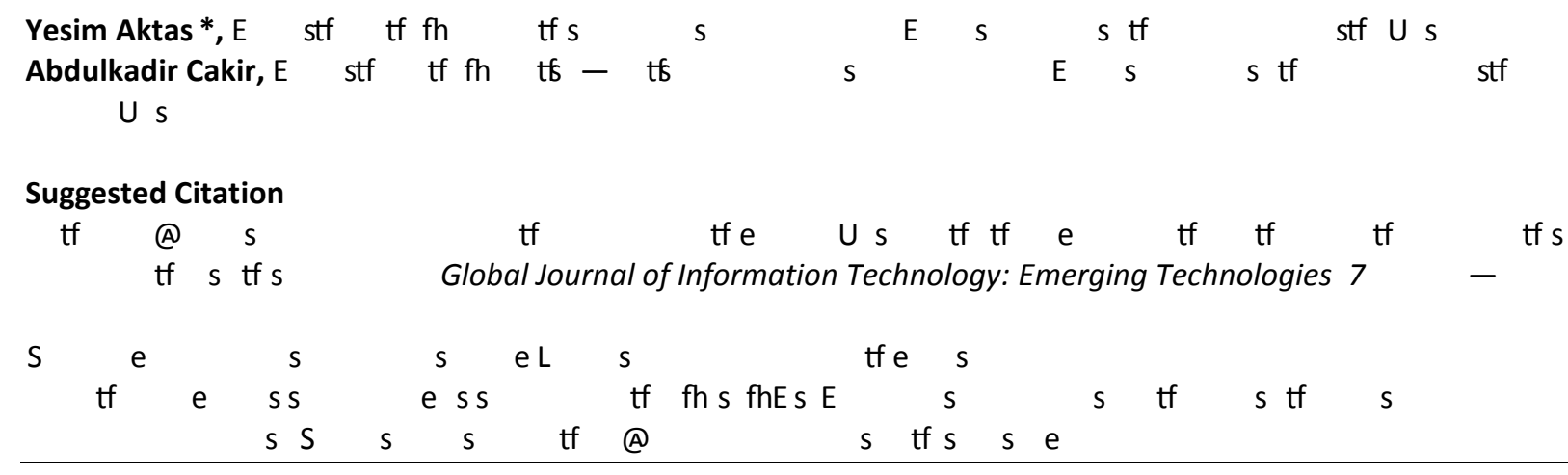

\section{Abstract}

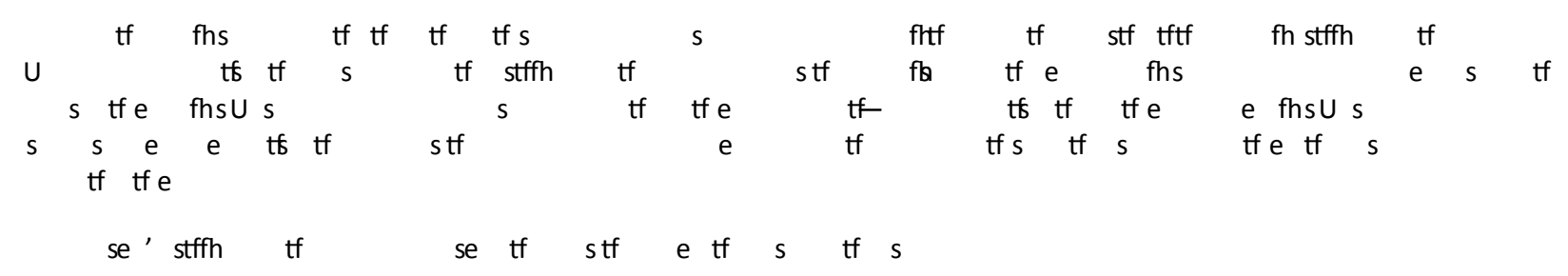

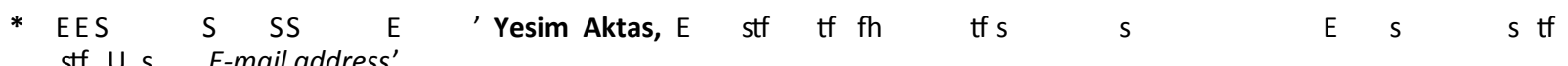


tf (A) $s$ tf $\quad$ tf $U s$ tf tf $e$ tf tf tf tf tf $s$ tf

Global

\section{Introduction}

$$
\begin{aligned}
& \text { tf } s \quad \text { tf } s
\end{aligned}
$$

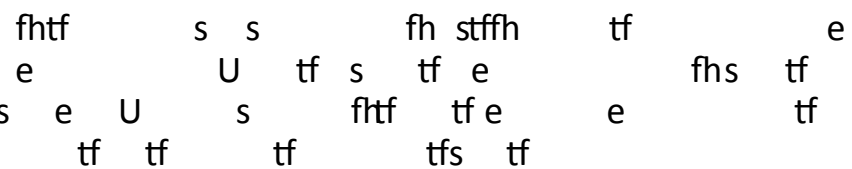

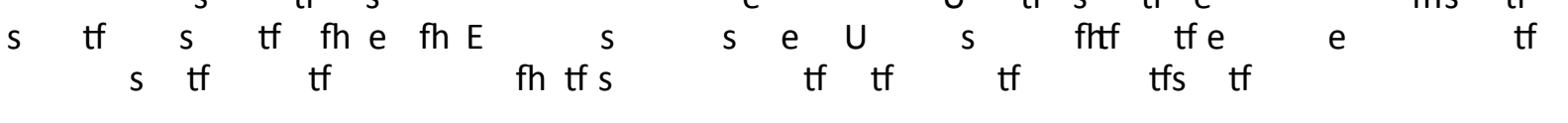

$$
\begin{aligned}
& \text { tf } \quad \text { tf } t f s \quad s \text { e e tf } \quad \text { tf } s \text { stf } s \text { s e tf } s \text { tf }
\end{aligned}
$$

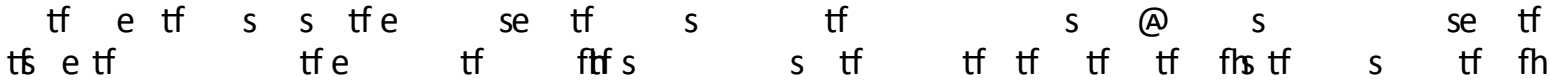

$$
\begin{aligned}
& \text { tf tf tf } s \text { e stf stf se tf } s \text { e e fhs }
\end{aligned}
$$

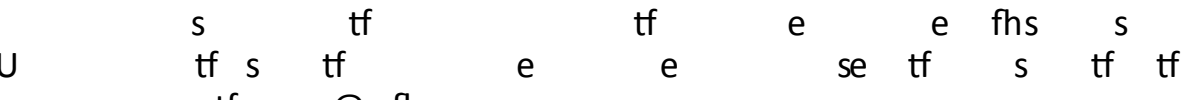

$$
\begin{aligned}
& \text { eUs tf } \text { (A) fh } s
\end{aligned}
$$

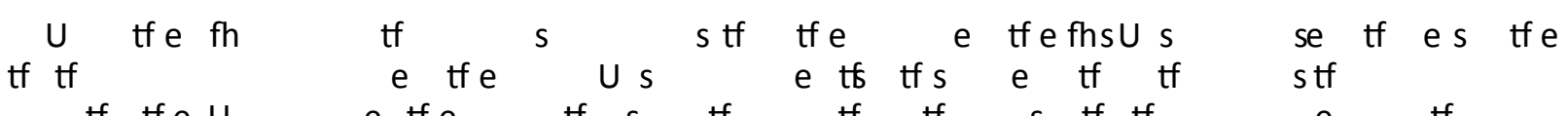

$$
\begin{aligned}
& \text { tf } t f e U \text { e tf e tf } s \text { tf } \text { tf tf } s \text { tf tf } \\
& \text { tf } e \text { tf } s \text { s tfe tf } s \text { tfe tf tf tffo } U s \text { se tfus } \\
& \text { fhs } t f \text { tf } U s \text { tf } e \text { tf } s \text { e tf } t \text { tf } s \text { U s } \\
& \text { tf } \quad \text { tf tf } s \text { tf } s \text { tf } s \text { eetf tf ee } s \text { tf@ fh } s
\end{aligned}
$$

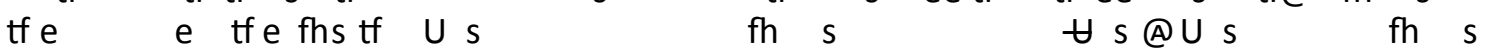

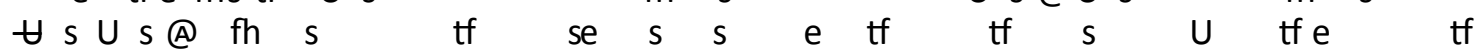

$$
\begin{aligned}
& s \text { tf tf se tf tfetf tf etf } s \text { tf } s \mathrm{~s} \text { tf }
\end{aligned}
$$

\section{Studies}

\subsection{Wordnet Anaysis Studies}

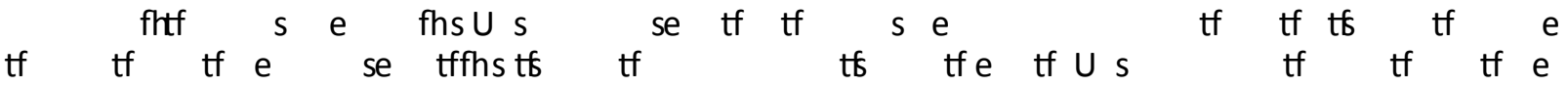
tf $t f$ fhs $s$ tf $t f$ tf $t f$ s tfe tf $s$ tf $s$ s e tf $t f$ tf $e$ tf $t f$ tf $s \quad$ e $U$ tf e s e e e tf tf

fh $s$ tf $s \quad s \quad s$ e tf e tf $s$ e stf e e

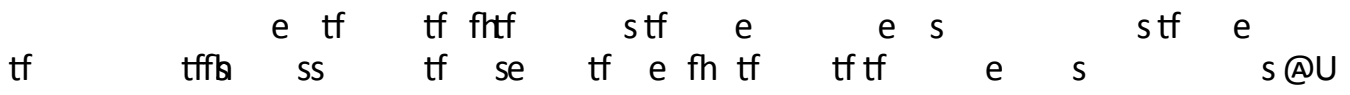

tf e s se e s tf tf $s$ tf fltf $U s$ tf se $s$ e e e tf eetf es tf $U$ e fltf $s$ tf $e \quad s \quad$ e se $s \quad s$ e stf stf $s$ tf $s$ tf $s \quad s \quad$ e tf $s \quad$ tfe ffh tf tf

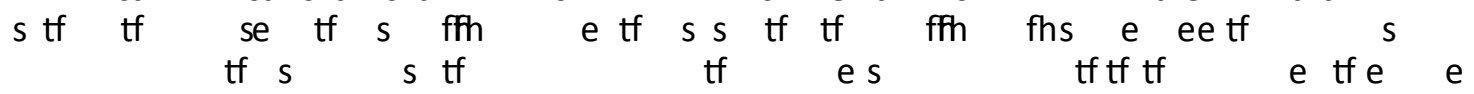

$s$ ss $s$ ss tf tf eetf es tf fltf $s \quad$ e tf s s $s \quad$ e

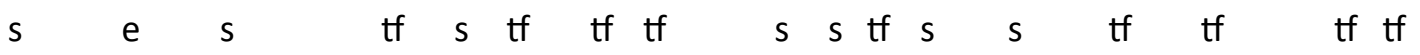

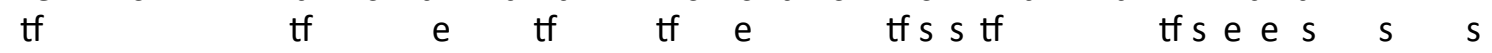
tf $E$ tf $s$ s $s$ fls tf $t f$ e tf $s$ tf

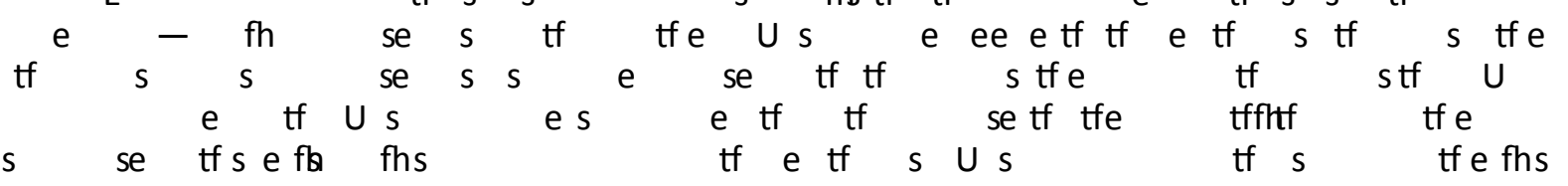


tf (A) S tf tf e U s tf tf e tf tf tf tf Journal of Information Technology: Emerging Technologies $7 \quad-$

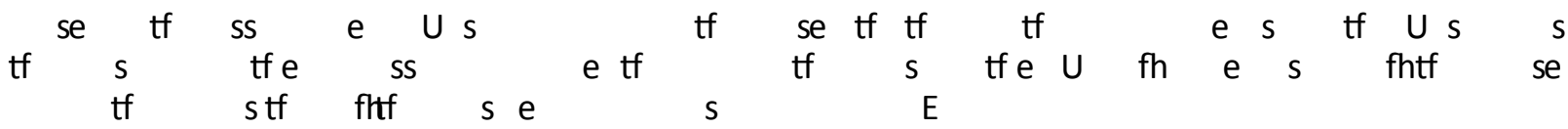

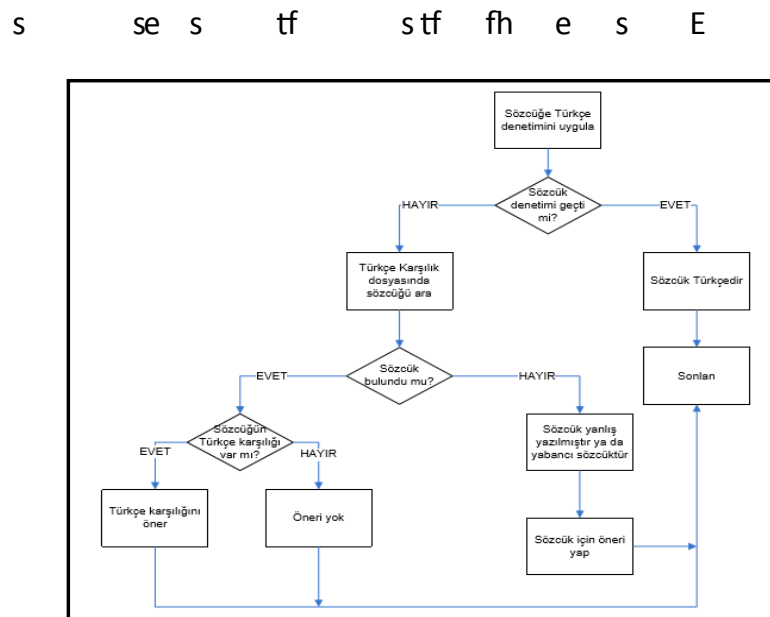

\subsection{Computer Network Terms And Resolution Recommedations}

$$
\begin{aligned}
& s \quad \text { tf e tf } \quad s \quad \text { fhtf } \quad \text { e } \quad \text { e tf } s \quad \text { e } \quad \text { tf } s \quad s e U U f h \\
& \text { U s se tf } U s \\
& \text { fh } J \text { U ths tf } t \text { fhs } t f \text { e tf } s \text { J } \\
& s \text { tf } t \text { e tf } s \text { tf e tf } s \text { s e tf tfe }
\end{aligned}
$$

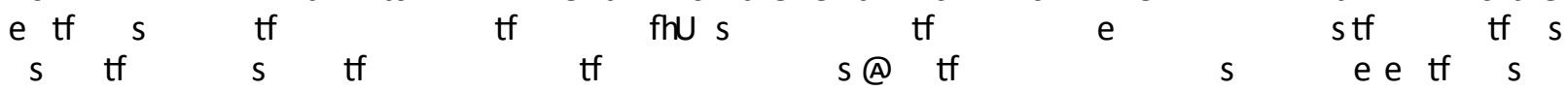

$$
\begin{aligned}
& \text { e se tf } s
\end{aligned}
$$

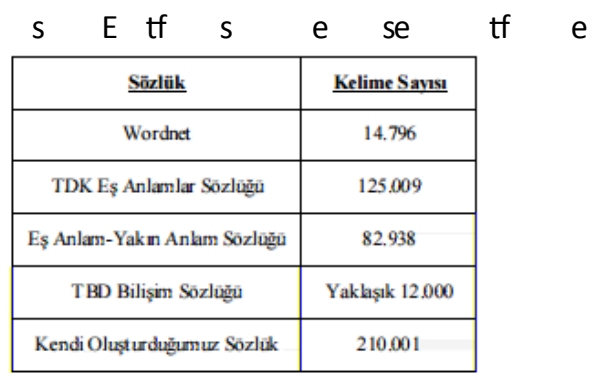

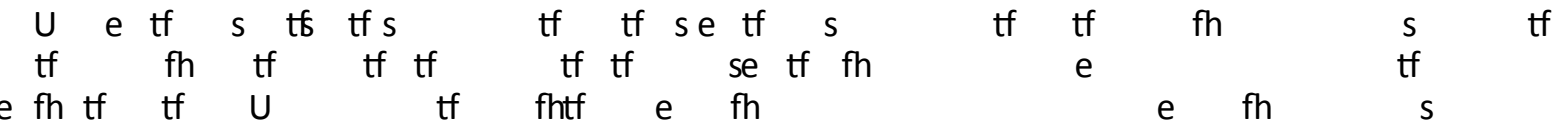

@ tf $s @$ tf @ tf $\mathrm{s} @ \mathrm{e} @ @$

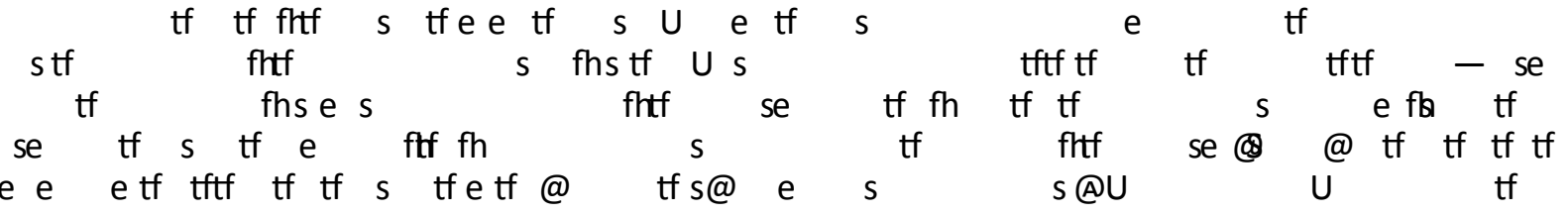




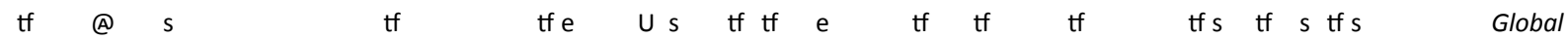
Journal of Information Technology: Emerging Technologies $7 \quad-\quad$

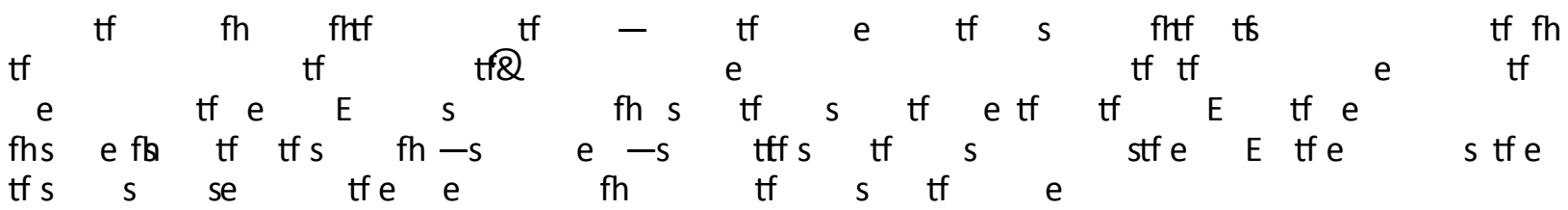
Q $s$ e eetf tf tf $s$ fhs tf tf fhs e tf $s$ fhe tf $s$ shs $U s$ tf $s$ tf tf $s$ $s$ e tf tf e e fh tf $\mathrm{e}$ tf $s$ fh tf tf $\mathrm{s}$ etf se tf $s$ e tf tf tf tf $s$ sfhs $\mathrm{SS}$ fh $\mathrm{tf}-\mathrm{e}$ tf tf tf

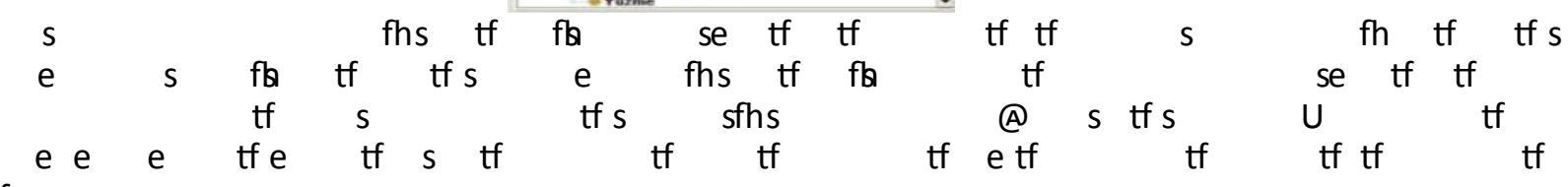

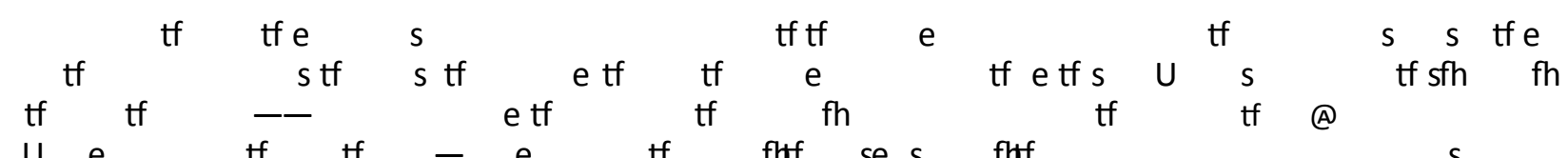

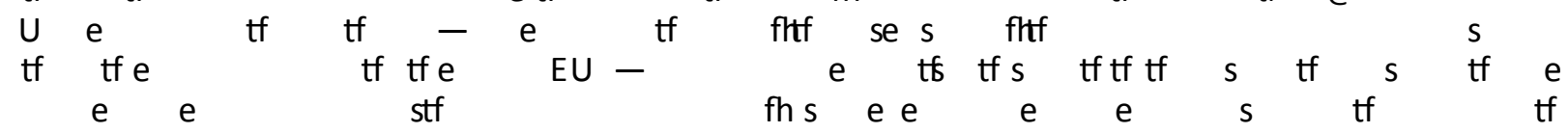
$\mathrm{e}$
fhs 
tf (A) S tf $\quad$ tf e $U \mathrm{~s}$ tf tf e tf tf tf tf tf $\mathrm{s}$ tf Journal of Information Technology: Emerging Technologies $7 \quad-$

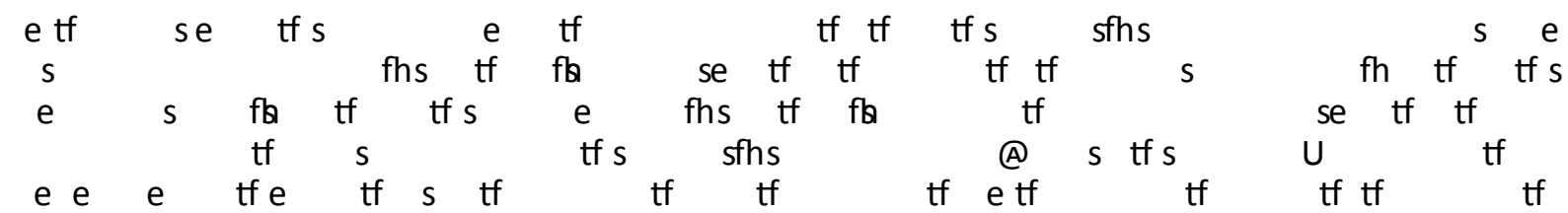
tf

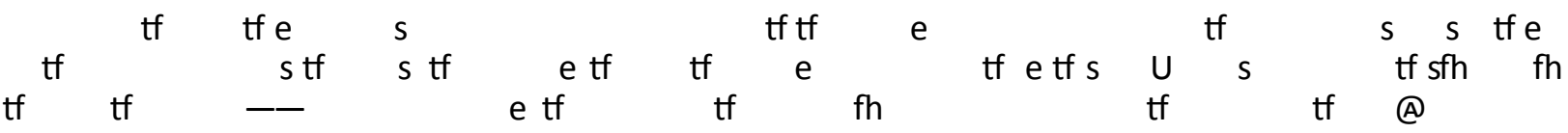
$U$ e tf $t f \quad$ e tf fhtf se $s$ fhtf

$s$ tf $t f e$ tf $t f e$ EU - J e tf tf $s$ tf tf tf $s$ tf $s$

tf $\mathrm{e}$ e e tf $\mathrm{stf}$ e e $\mathrm{e}$ e $s$ tf tf fhsU s $s$ tf $\mathrm{e}$ (A) fh $s$

\section{Results and Recommendations}

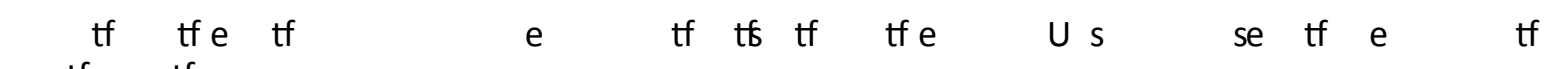
$s$ tfe e tf $s$ s e

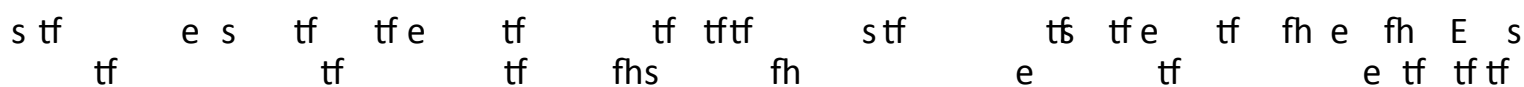
e fh tf ffh tf e e e
tf $\quad$ e $e_{\text {tf }}$ tf $\mathrm{s}$
e fhs tf fhs
tf $s$ tfe tf $s$ fh tf $s$ e tf $s$
tf $\quad$ tf e tf
$s$ tfeUs

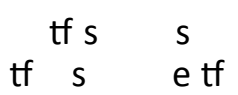
e fh ${ }_{\text {tf }}^{\text {stfh }}$ stf
stf $\mathrm{e}$
tf fhs fhs
tf $s$

\section{Acknowledgments}

$U$ tf $s$ s $s$ tf fh tf

$s$ e e fh

stf $\quad$ tf $t f \quad s \quad t f$

s e

- tftf tf fltf $s$

\section{References}

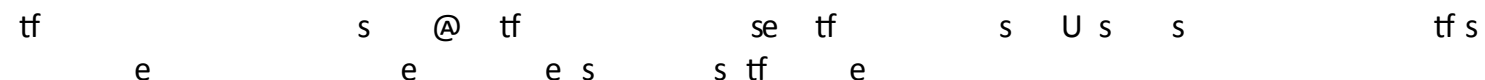

$$
\begin{aligned}
& s \text { tf } \mathrm{US} \text { se tf tf tf tf } \mathrm{s} \text { s e jej } U
\end{aligned}
$$

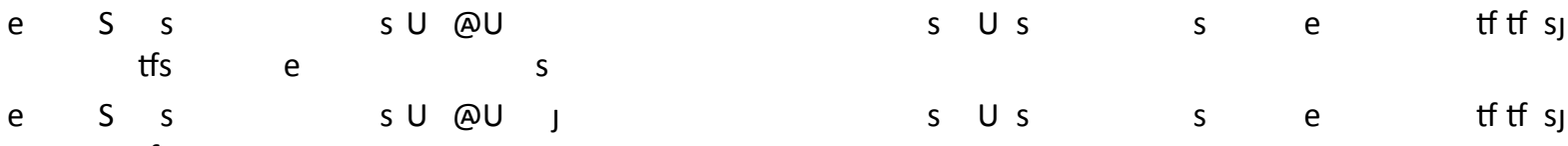

$$
\begin{aligned}
& \text { e } S \underset{t f s}{S} \quad \text { e } s U \text { @U } \\
& \text { tf } \quad \text { @ fh } \mathrm{s} \text { e se tf } \mathrm{sU} s \text { Romanian Journal of Information }
\end{aligned}
$$

$$
\begin{aligned}
& \begin{array}{r}
\text { Science and Technology, } 7- \\
\text { s tf }
\end{array} \\
& \text { U S tf } \\
& \text { U S H } S \text { E tf } \\
& \text { U If }
\end{aligned}
$$




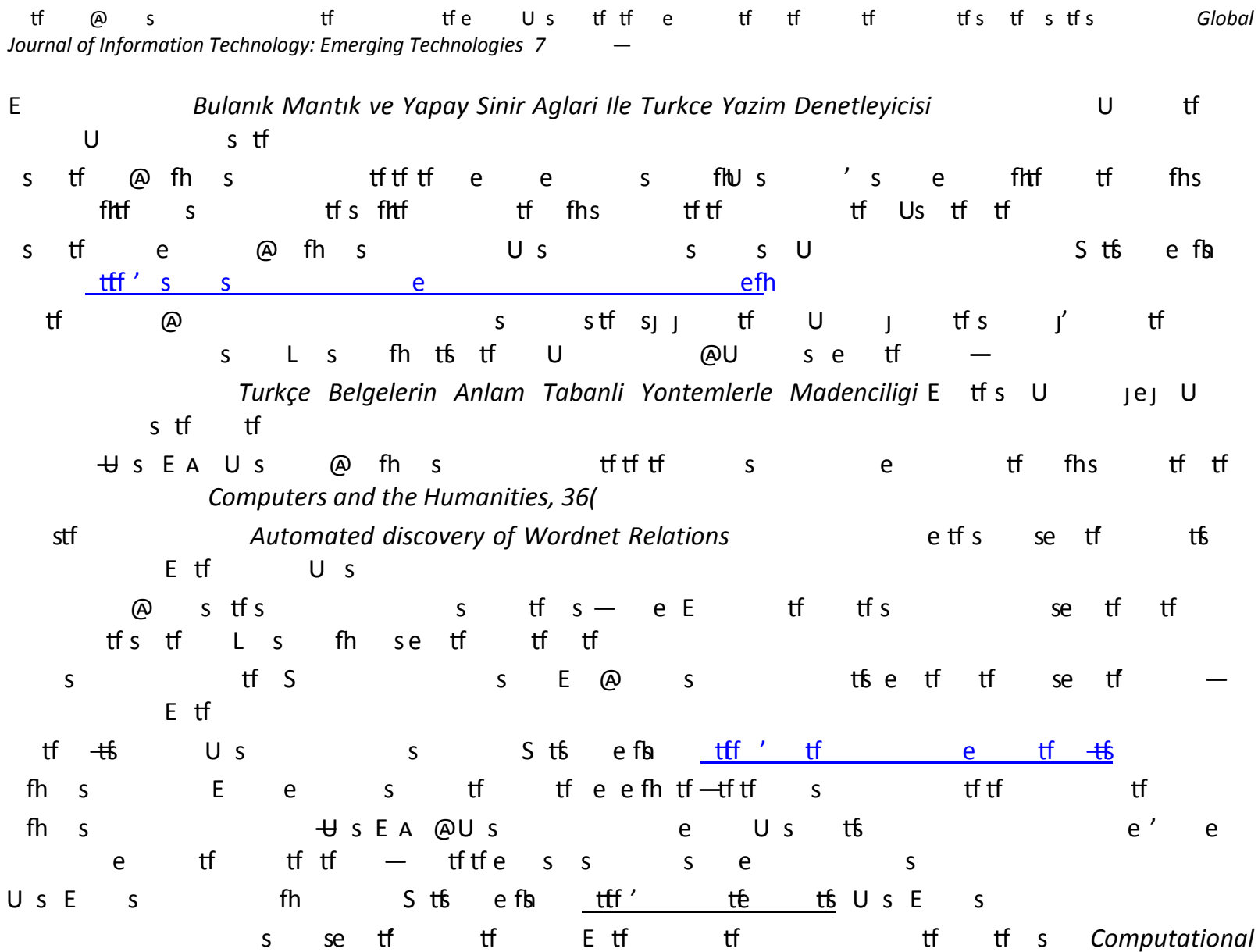
Linguistics, 25 